\title{
«Cela vaut la peine de prendre grand soin du système de santé»
}

\author{
Le système de santé suisse est un secteur économique en pleine croissance. Mais les \\ dépenses ne cessent, elles aussi, d'augmenter comme en témoignent les hausses \\ annuelles de cotisations. Reiner Eichenberger, Professeur d'économie politique à \\ I'Université de Fribourg, nous explique où il entrevoit des potentiels d'amélioration.
}

Interview:

FMH Communication
Quelle est, selon vous, l'importance économique du système de santé suisse?

Reiner Eichenberger: Le système de santé suisse représente près de $11 \%$ du PIB. Mais cela ne veut pas dire grand-chose. De nombreux secteurs économiques ont une importance comparable si la définition qu'on en donne est suffisamment large. Il n'en reste pas moins que le système de santé est un employeur majeur qui génère une part importante de la plus-value. Si on le foule aux pieds, une partie de cette plus-value disparaît. Cela vaut donc la peine de prendre grand soin du système de santé.

Un aspect intéressant est le rapport qu'entretiennent de nombreux citoyens et politiciens avec ce secteur: ils se plaignent quand il croît alors qu'ils réclament une croissance continue pour la plupart des autres branches. Or la croissance peut également être cision politique, il n'existe aucune incitation à économiser. Le patient ne paie qu'une petite partie des prestations sollicitées. Le fournisseur de prestations tire un bénéfice à prescrire une prestation, ce qui est un incitatif clair. Et le processus de décision politique est déterminé par les groupes d'intérêts suivants: médecins, hôpitaux, industrie pharmaceutique, pharmaciens, assureurs et organisations de patients, qui tous profitent du niveau élevé des prestations et des coûts. Les assureurs gagnent, en effet, davantage si les coûts, et donc les primes, sont élevés. Les organisations de patients ont tendance à représenter des malades qui veulent des prestations avant de vouloir des économies. Par contre, personne ne défend les intérêts des citoyens en relativement bonne santé - à savoir un bon rapport qualité-prix. Ce qui explique pourquoi le système ne cesse d'enfler.

\section{Médecins, hôpitaux, industrie pharmaceutique, pharmaciens, assureurs et organisations de patients - tous profitent du niveau élevé des prestations et des coûts}

une bonne chose pour le système de santé. En fin de compte, tout le monde s'accorde à dire que la santé est notre bien le plus précieux. Les problèmes n'apparaissent que si la croissance s'accompagne d'une augmentation de l'inefficacité.

Quelles sont, selon vous, les sources d'inefficacité? Que ce soit au niveau des bénéficiaires de prestations, des fournisseurs de prestations ou du processus de dé-

\section{Portrait}

Reiner Eichenberger est Professeur ordinaire d'économie politique à I'Université de Fribourg et directeur de recherches au CREMA (Center for Research in Economics, Management, and the Arts). L'analyse économique des processus politiques et des institutions politiques est l'un de ses domaines de prédilection.

\section{Le prix a-t-il perdu sa signification?}

Oui, complètement. La situation est comparable à la surexploitation de l'environnement ou à un abonnement général des transports publics. Les usagers supportent les coûts occasionnés de manière forfaitaire et non dans leur intégralité. Ceci conduit logiquement à une problématique de pénurie puisque chaque prestation génère un bénéfice sans toutefois en avoir le prix. C'est à peu près la même chose dans le système de santé. Le patient ne paie qu'une petite partie du prix. C'est pourquoi il revendique le meilleur traitement - souvent le plus onéreux afin de limiter à un minimum ses frais effectifs en cas de maladie tels qu'effets secondaires, durée de guérison ou risques. En Suisse comme dans la plupart des autres pays, il n'existe aucune incitation à orienter le progrès technique vers l'économie de coûts. 
L'éthique et l'économicité se contredisent-elles?

Le système de santé connaît une importante redistribution que beaucoup appellent «solidarité». Dans le cas le plus simple, ceci signifie que les primes devraient être les mêmes pour tous, indépendamment de la consommation de prestations. Ceci mène inéluctablement à une confusion et aux effets connus comme la sélection des risques par les caisses-maladie.

\section{Un modèle éthique consisterait à assurer des risques que le citoyen normal ne peut pas supporter}

D'un point de vue économique, la solidarité au sein du système de santé est-elle judicieuse?

D'un point de vue économique, la solidarité n'est bien évidemment pas une mauvaise mais une bonne chose. Néanmoins, la redistribution peut être efficace ou inefficace. Si on procède à une redistribution par une aide aux objets, on crée non seulement une distorsion des prix mais on génère aussi d'énormes coûts économiques. La bonne méthode serait l'aide aux personnes, en l'occurrence des versements directs à ceux que l'on souhaite subventionner. De nombreux problèmes inhérents au système de santé proviennent du fait qu'il sert de dispositif de redistribution de la population jeune vers la population plus âgée: les premiers versent en moyenne d'énormes cotisations et les derniers sont subventionnés. Si l'on souhaite une telle redistribution, il faut l'assumer et introduire un «impôt jeunes», mais ne pas se servir du système de santé pour y parvenir.

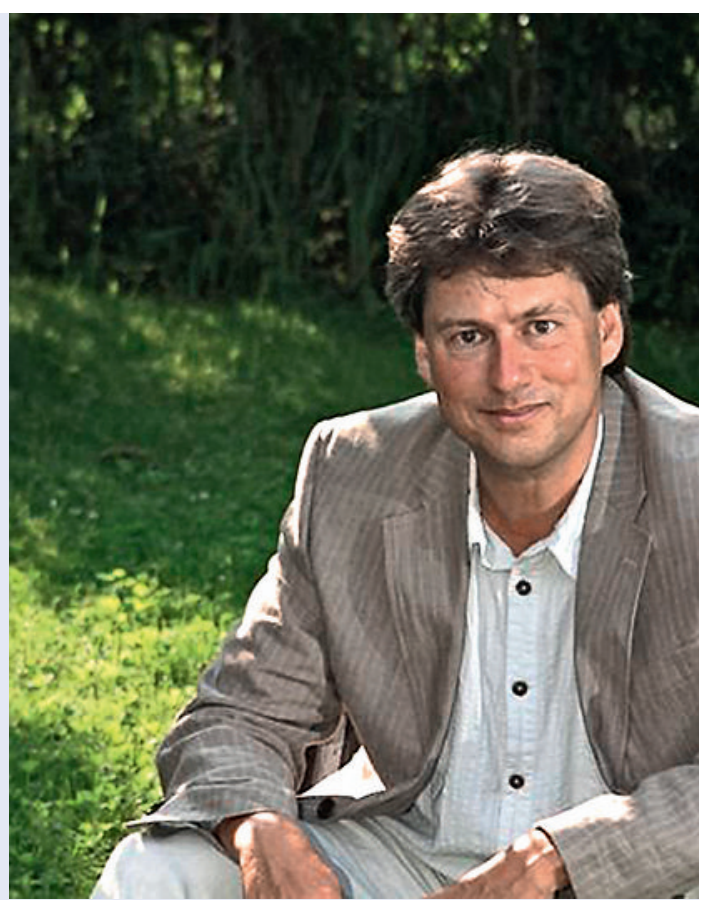

Reiner Eichenberger est Professeur ordinaire d'économie politique à l’Université de Fribourg.
Où sont les potentiels d'économie?

Nous avons un système non éthique dans lequel le citoyen normal est assuré pour un risque normal. Les assurances n'ont pas vocation à assurer des actes médicaux auxquels chacun recourt de temps à autre. Ceci est totalement insensé du point de vue des coûts. On retire au patient toute incitation à économiser et instaure une énorme bureaucratie pour la facturation. Un modèle éthique, en revanche, consisterait à assurer des risques que le citoyen normal ne peut pas supporter. Dans une société qui fonctionne normalement, un père de famille de plus de 30 ans doit avoir des économies lui permettant d'assumer lui-même de petits risques comme la grippe par exemple. Les factures médicales peuvent apparaître élevées sur le moment. Mais si on les compare à une facture d'électricien ou aux dépenses faites dans les restaurants ou en vacances, il s'agit bien souvent de montants dérisoires. Néanmoins, on pense généralement que tout doit être assuré. Ceci mène à une explosion des coûts car le citoyen sollicite un nombre démesuré de prestations, les assureurs ont d'énormes frais et même les médecins se voient privés de toute incitation à économiser.

\section{Est-ce là une critique à l'assurance obligatoire?}

Pour les bénéficiaires de revenus normaux, l'assurance obligatoire comporte des obligations inutiles: par exemple, il nous faut des franchises de base beaucoup plus élevées. Cette mesure exclurait bien évidemment les jeunes qui n'ont pas encore eu la possibilité d'économiser. Mais un système éthique implique également d'assumer une certaine responsabilité personnelle. Un modèle qui permettrait d'économiser pour les dépenses de santé avec une exonération d'impôts est également envisageable. Il est incompréhensible que tout un modèle étatique soit conçu de telle sorte que des adultes, toujours à la limite financièrement parlant, ne puissent plus assumer eux-mêmes les coûts du moindre petit risque.

Dans la politique nationale, plus de 200 motions de politique sanitaire sont en suspens, dont une grande partie concerne le volet des coûts. Les politiciens sous-estimentils le potentiel économique du système de santé?

La plupart des tentatives consiste à fixer un plafond global. Mais cela ne sert à rien si on ne combat pas l'inefficacité. En grande partie, la croissance des coûts est très simple à expliquer. Les hausses de 2 à $5 \%$ des coûts de la santé, régulièrement publiées, sont souvent de la désinformation. Elles ne tiennent pas compte de la croissance démographique actuelle de 1 à 1,4\% ni d'une inflation moyenne de $1 \%$. Sans ces deux facteurs, les coûts continuent d'augmenter de 0 à $3 \%$ selon l'année. Si l'on tient compte, en outre, d'une croissance moyenne réelle des revenus de $1 \%$ par tête et du fait que la santé est pour de nombreuses personnes le bien le plus précieux, l'augmentation des coûts n'est tout d'un coup plus aussi dramatique. La santé est typiquement ce qu'on appelle un «bien supé- 
rieur»: la demande envers de nombreux biens supérieurs est sur-proportionnelle à l'augmentation de la richesse. Ce qui relativise considérablement la forte croissance des coûts. Les citoyens sont véritablement attachés aux prestations de santé comme le montrent diverses votations. Non pas parce qu'ils n'ont pas conscience des coûts, mais parce que la santé leur tient particulièrement à cœur. Aujourd'hui, toutefois, la politique discute davantage du plafond global que de l'économicité. C'est là une mauvaise approche. Certes, les coûts baissent, mais également la qualité des prestations. Le citoyen suisse qui, heureusement, a son mot à dire, ne souhaite pas cela.

Une plus grande concurrence est-elle la clé d'une baisse des coûts?

La concurrence débouche sur une diminution des coûts par unité de prestation standardisée. Néanmoins, ou justement pour cette raison, elle peut également conduire à une hausse des coûts globaux. Quand l'efficacité augmente et les prestations s'améliorent grâce à la concurrence, la disposition à payer, et donc la demande, augmentent également. Comparez cela à l'industrie informatique: de plus en plus d'argent est dépensé à travers le monde pour les ordinateurs; non pas en raison de leur plus mauvaise qualité ou de leur prix plus élevé, mais parce qu'ils sont de plus en plus performants et de moins en moins chers. C'est là une croissance économique normale dans un secteur donné, générée par une augmentation de l'efficacité.

La qualité des prestations de santé en Suisse est très élevée. Pourquoi ne sont-elles pas exportées?

Cela a un rapport avec les interventions étatiques. Les hôpitaux n'ont aucune incitation à attirer des patients en Suisse puisqu'ils sont en grande partie subventionnés. Parallèlement, les patients disposent le plus souvent d'assurances qui ne prennent en charge que les traitements dispensés dans leur propre pays. Ils n'ont donc aucun intérêt à se faire soigner à l'étranger. Certains domaines, cependant, comme la médecine de pointe, la médecine dentaire ou des interventions soumises à des réglementations très différentes d'un pays à l'autre telle que l'insémination artificielle, sont plus ouverts et connaissent un certain «tourisme». Cette tendance va augmenter à l'avenir. Les patients deviendront plus mobiles et devront prendre davantage de coûts à leur charge, ce qui les incitera à solliciter les offres étrangères de qualité.

Doit-on mettre les bénéficiaires de prestations davantage à contribution et déléguer une partie de la responsabilité? Ou cela conduit-il à une médecine à deux vitesses?

Le débat sur la médecine à deux vitesses est ridicule. Aujourd'hui déjà, nous connaissons une médecine à plusieurs vitesses avec des différences dramatiques: l'offre de prestations diffère fortement en fonction du lieu de résidence. Par ailleurs, les techniques utilisées par les médecins et les hôpitaux varient considérablement selon la personne, l'institution ou la région. Toutes ces inégalités se reflètent ensuite dans la grande disparité des coûts par assuré: plus du double à Genève qu'en Appenzell. A cela s'ajoutent d'énormes différences en matière de santé et d'espérance de vie selon la catégorie professionnelle et la tranche d'âge. C'est donc complètement abstrus de s'opposer à toute réforme en arguant le maintien d'une médecine à une vitesse.

Selon vous, quel est le défi à relever au niveau politique? Maints politiciens méritent la compassion. Ils sont prisonniers d'une camisole de force. Ils sont mués par les incitations qui leur sont prescrites par les groupes d'intérêts parfaitement organisés. Comme tous ces groupes sont attachés à des prestations et des coûts de haut niveau - comme je l'ai évoqué plus haut -, rares sont les politiciens qui défendent des mesures d'économie sérieuses et l'amélioration de l'efficacité.

Cela contredit les nombreuses motions visant à réduire les coûts au bénéfice des assurés.

Malheureusement, ces motions ne sont souvent que de la poudre aux yeux. De nombreux politiciens disent tout simplement ce que l'électeur moyen veut entendre. Et dans les Chambres, ils décident de tout autre chose, à savoir de ce que veulent les groupes d'intérêts bien organisés. Le simple électeur ne peut pas contrôler ce qui se passe véritablement au Parlement. En revanche, les groupes d'intérêts bien organisés sont parfaitement en mesure d'estimer le comportement des politiciens et les objectifs qu'ils peuvent atteindre. Cela leur permet d'exercer une plus grande influence.

N'en déplaise aux politiciens qui préféreraient, bien évidemment, être aux commandes plutôt que de se laisser commander. Mais heureusement, il y a des exceptions même parmi les politiciens.

Quel message adressez-vous aux médecins?

L'important pour les médecins est de retrouver confiance en soi. Le préjugé selon lequel la hausse des coûts résulterait des salaires élevés des médecins se base sur une erreur de raisonnement. Une telle situation impliquerait une augmentation massive et constante des salaires. Or ce n'est pas le cas. D'un autre côté, il faut plus de transparence sur les prestations de santé et leur qualité. Certaines personnes affirment que nous avons le meilleur système de santé. D'autres disent qu'il n'est pas si performant que cela. Il convient d'effectuer des comparaisons claires et de révéler les points faibles afin de procéder à des améliorations dans ces domaines. 